\title{
AGR-5/6/7 Irradiation Summary as of the End of Cycle 164B
}

\author{
Joe Palmer
}

April 2019

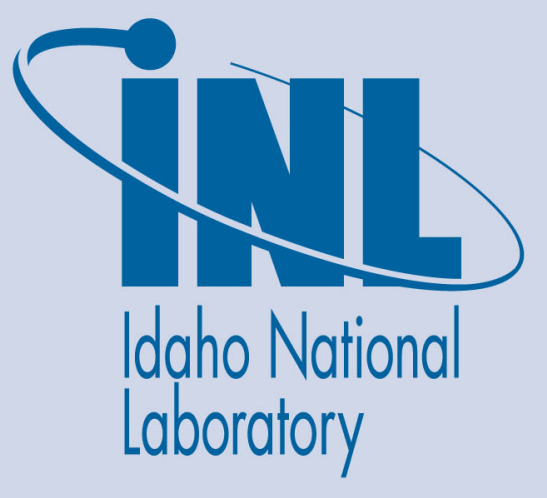

The INL is a U.S. Department of Energy National Laboratory operated by Battelle Energy Alliance 


\title{
AGR-5/6/7 Irradiation Summary as of the End of Cycle 164B
}

\author{
Joe Palmer
}

April 2019

Idaho National Laboratory Idaho Falls, Idaho 83415

http://www.inl.gov

Prepared for the

U.S. Department of Energy

Under DOE Idaho Operations Office

Contract DE-AC07-05ID14517 


\title{
AGR-5/6/7 Irradiation Summary as of the End of Cycle 164B
}

\section{Technical Coordination Team \\ April 9, 2019}

\author{
Joe Palmer \\ Mechanical Engineer, PE
}

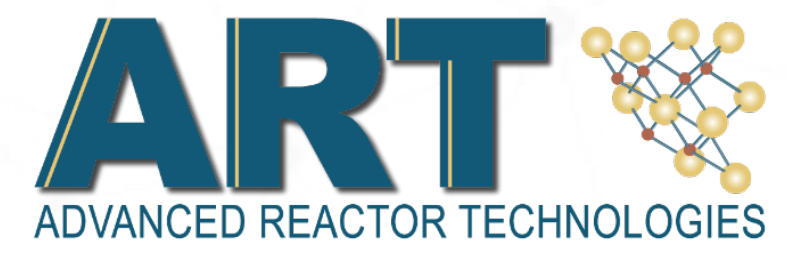




\section{Thermocouple Performance}

\begin{tabular}{|l|r|r|r|r|r|r|}
\hline $\begin{array}{l}\text { Survivors by } \\
\text { Capsule }\end{array}$ & $\begin{array}{l}\text { End of } \\
\text { Assem }\end{array}$ & $\begin{array}{l}\text { End of } \\
\text { Install }\end{array}$ & $\begin{array}{l}\text { End of } \\
\text { 162B }\end{array}$ & $\begin{array}{l}\text { End of } \\
\text { 163A }\end{array}$ & $\begin{array}{c}\text { End of } \\
\text { 164A }\end{array}$ & $\begin{array}{c}\text { End of } \\
\text { 164B }\end{array}$ \\
\hline Cap 5 & 4 & 3 & 3 & 3 & 3 & 3 \\
\hline Cap 4 & 5 & 4 & 4 & 4 & 4 & 4 \\
\hline Cap 3 & 15 & 13 & 12 & 12 & 9 & 9 \\
\hline Cap 2 & 8 & 8 & 8 & 8 & 6 & 3 \\
\hline Cap 1 & 17 & 17 & 11 & 10 & 7 & 4 \\
\hline $\begin{array}{l}\text { Total } \\
\text { Surviving }\end{array}$ & 49 & 45 & 38 & 37 & 29 & 23 \\
\hline
\end{tabular}




\section{AGR-5/6/7 TC Performance Compared to Past AGR Tests}

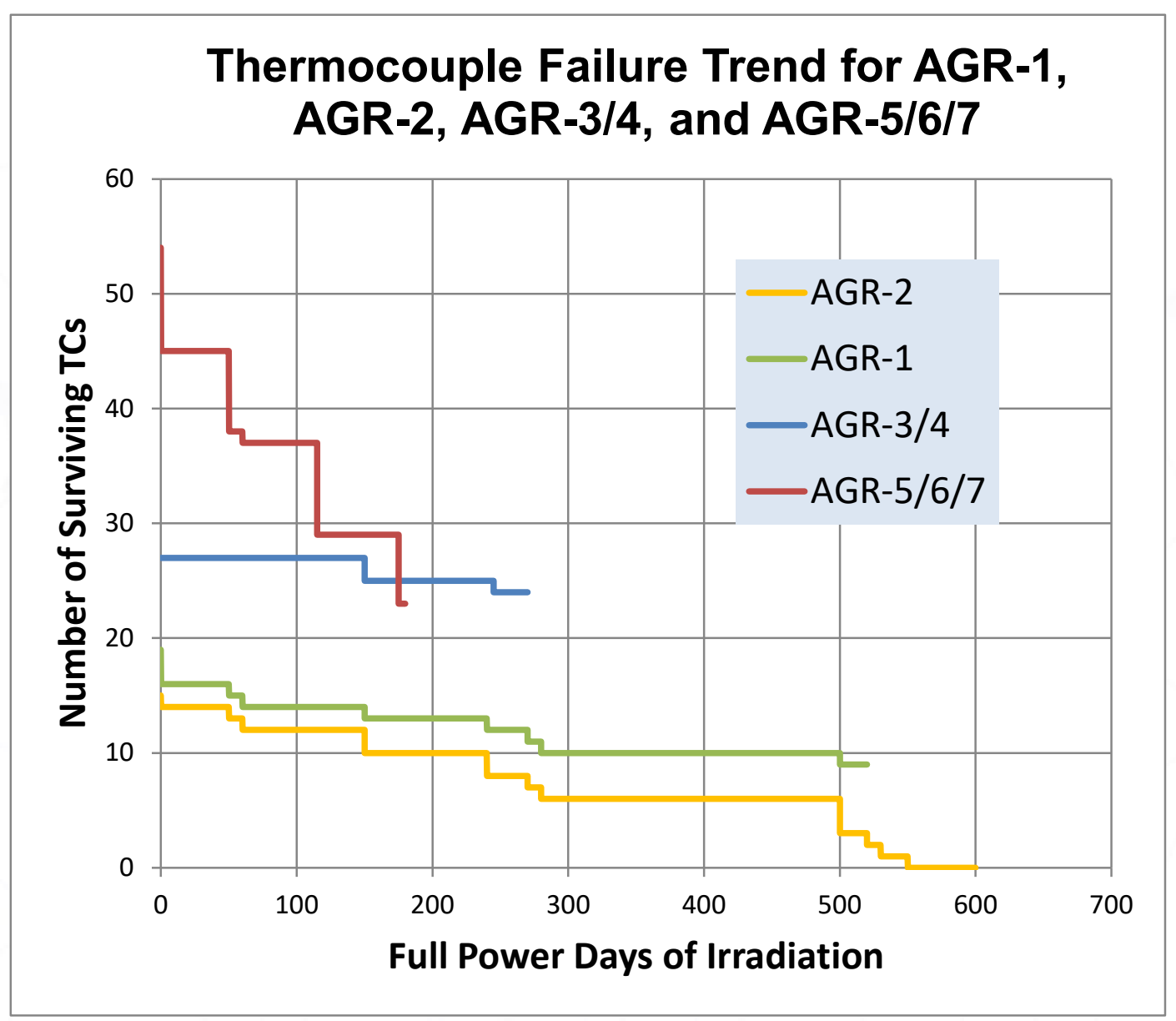




\section{Thermocouple Performance (cont)}

- The AGR-5/6/7 thermocouple failure rate has been high

- Project that all thermocouples in Capsules 1 and 2 will fail prior to completion of irradiation

- AGR-1 and AGR-2 also experienced high failure rates with 3 of the 6 capsules in each experiment having no functioning thermocouples for more than half of the irradiation period. 


\section{AGR-5/6/7 Compacts Daily Heat Rate}

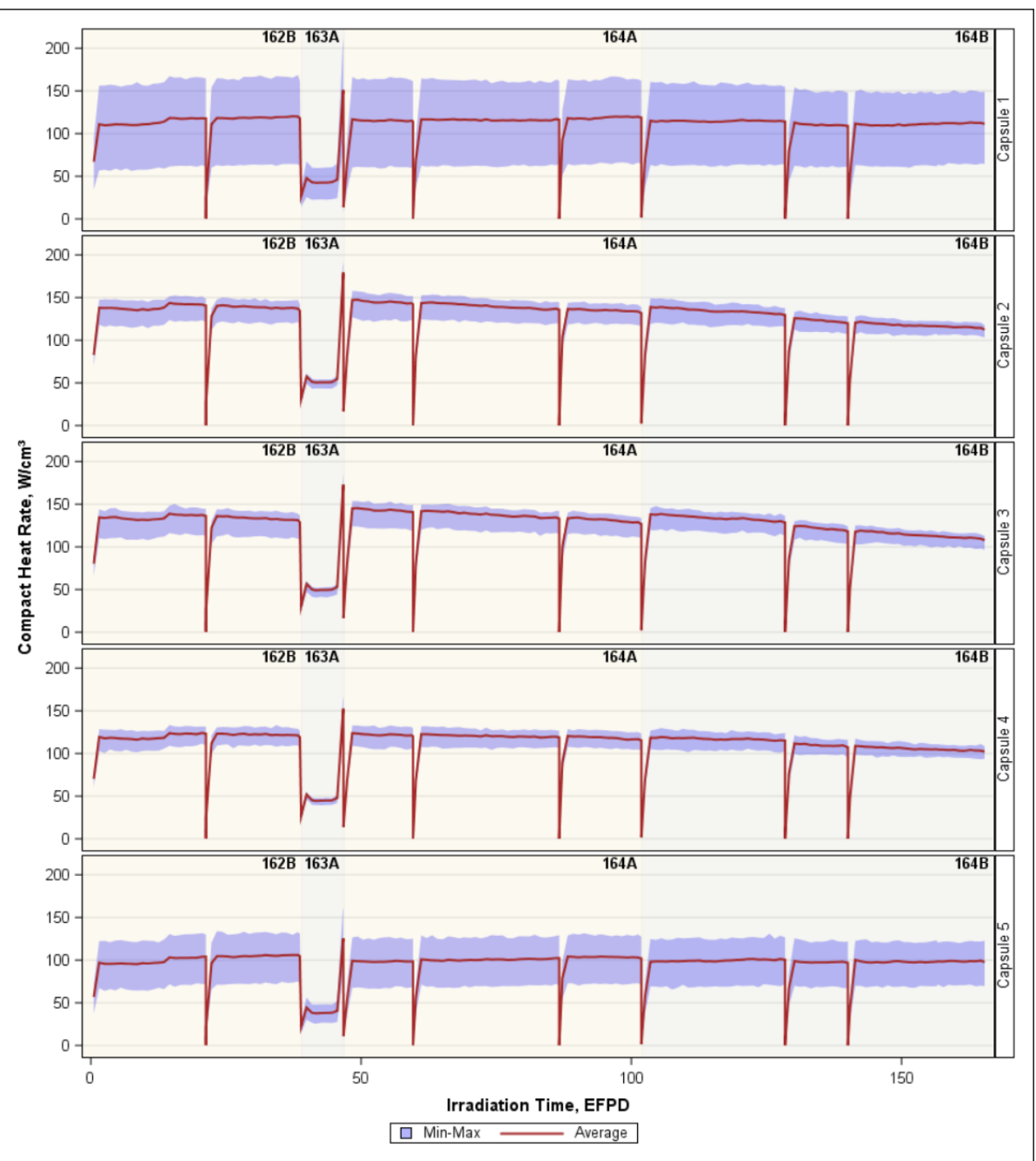




\section{AGR-5/6/7 Fuel Particle Temperatures}

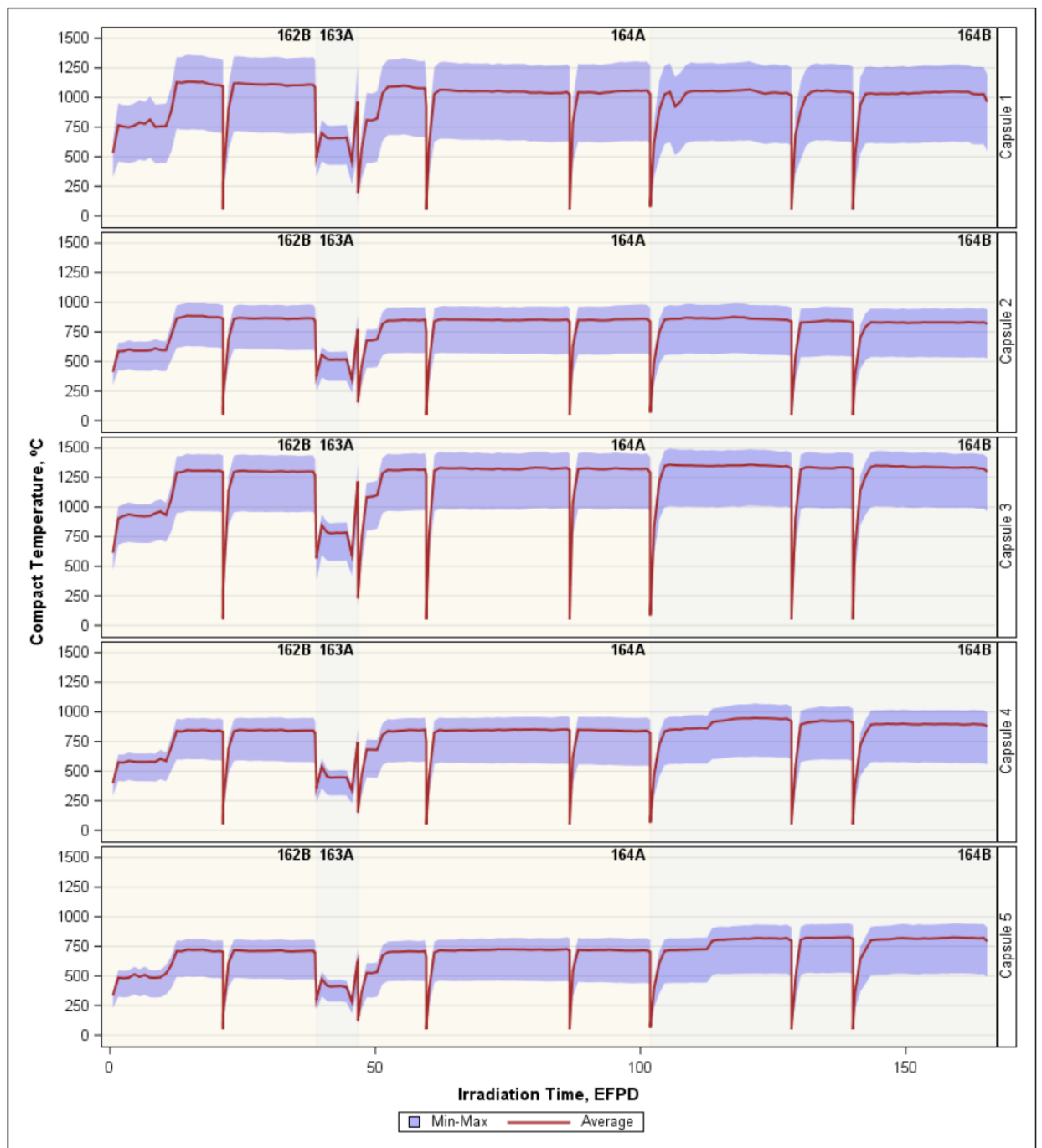




\section{Instantaneous AGR-5/6 Fuel Temperature Range Distribution}

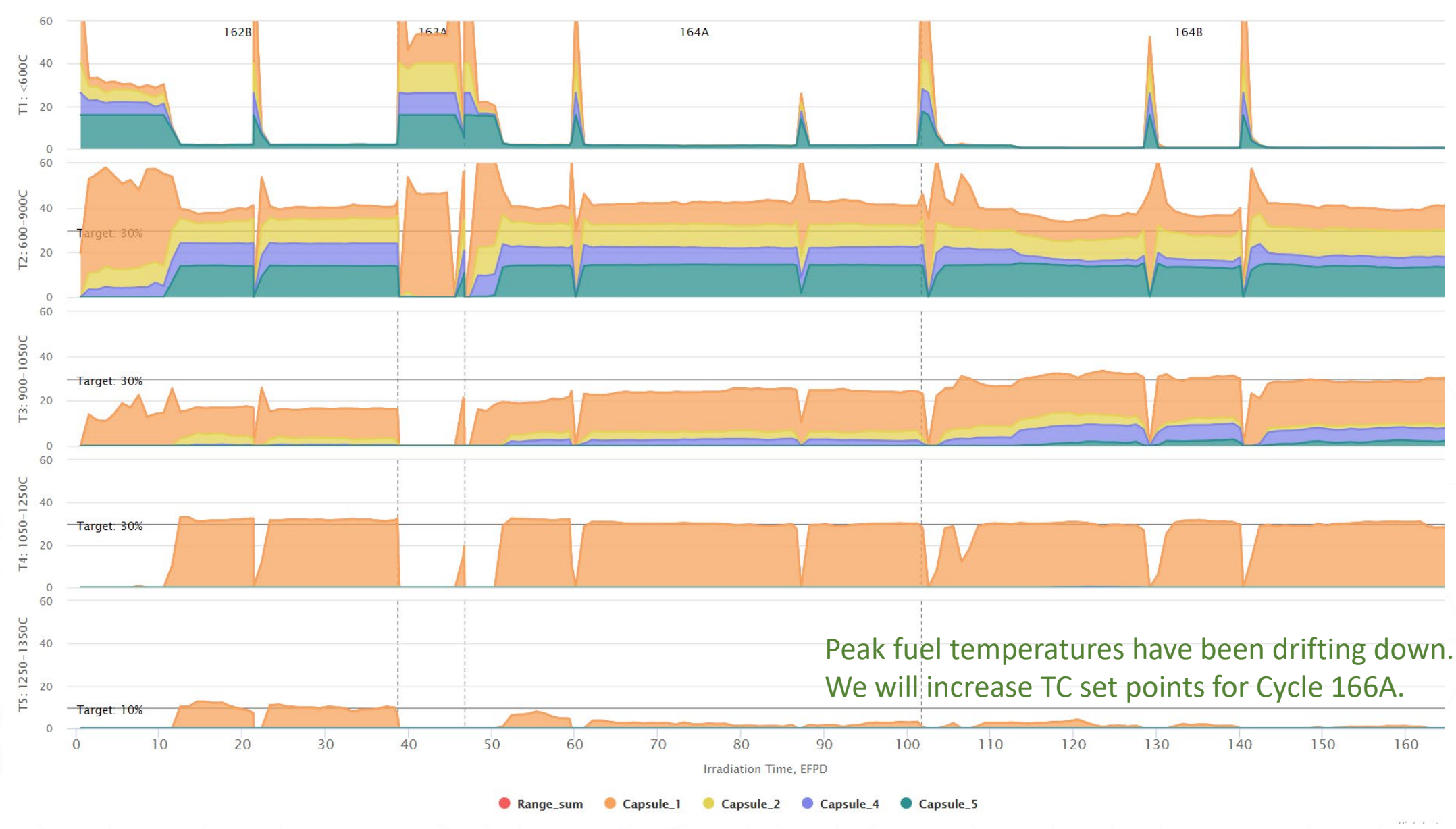




\section{Instantaneous AGR-7 Fuel Temperature Range Distribution}

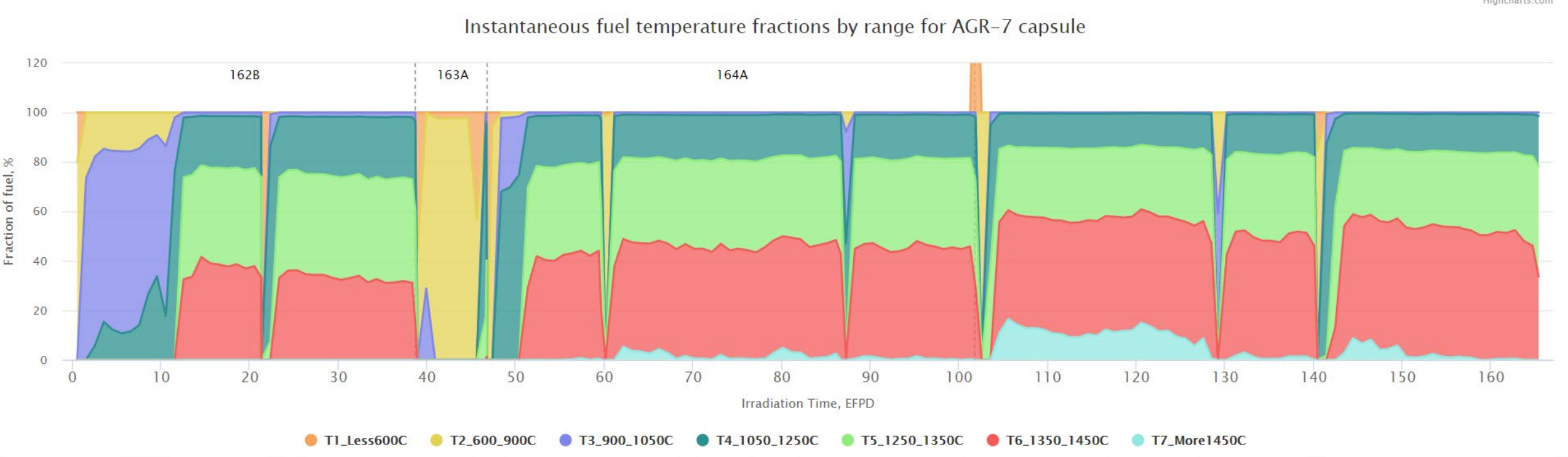




\section{T-Avg V-Avg AGR-5/6 Fuel Temperature Range Distribution}

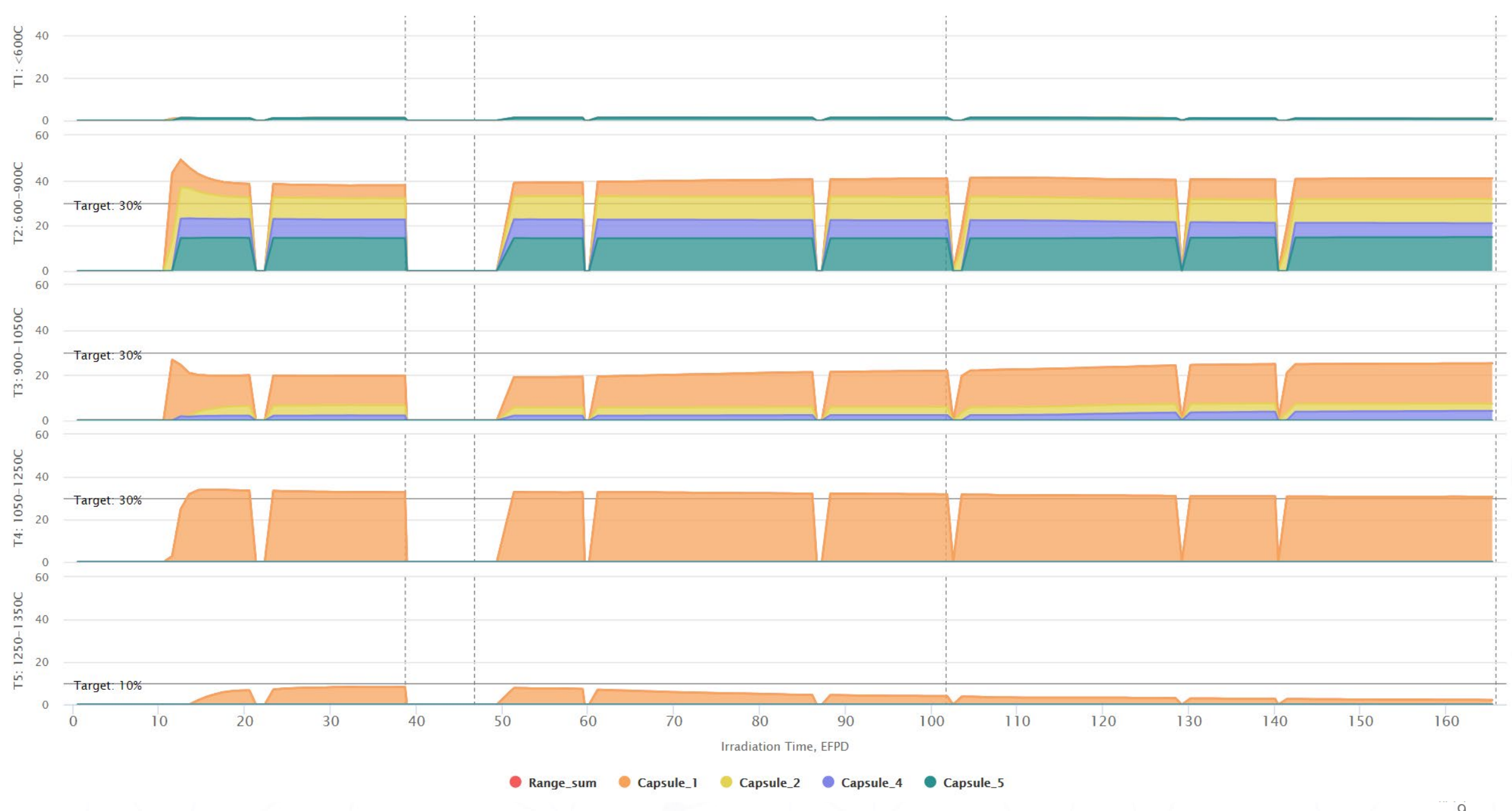




\section{T-Avg V-Avg AGR-7 Fuel Temperature Range Distribution}

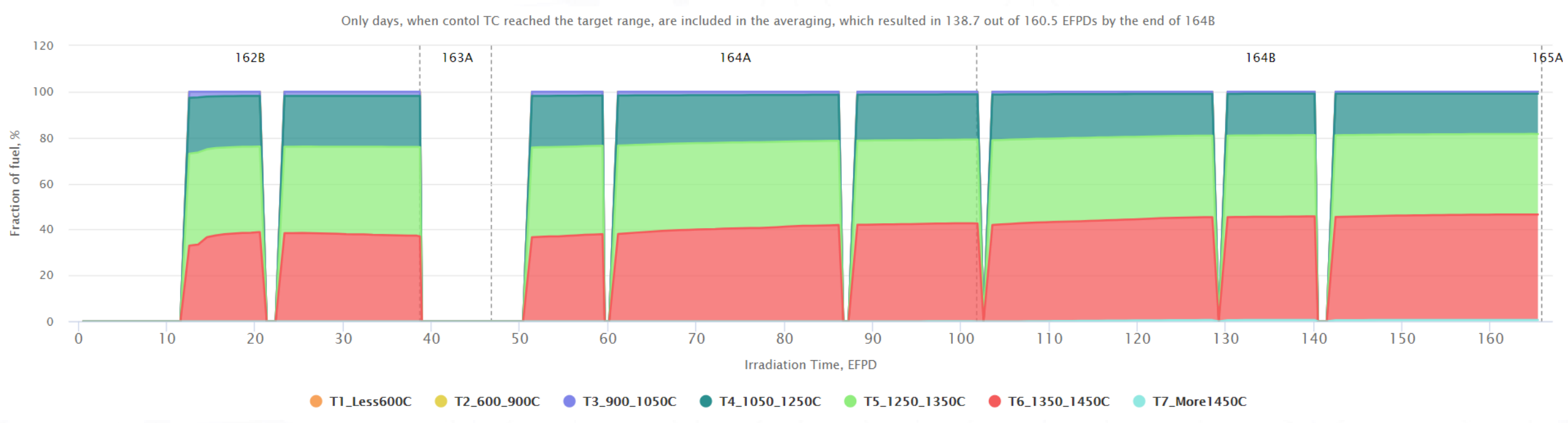




\section{Percent Burnup for Capsules 2 and 3}

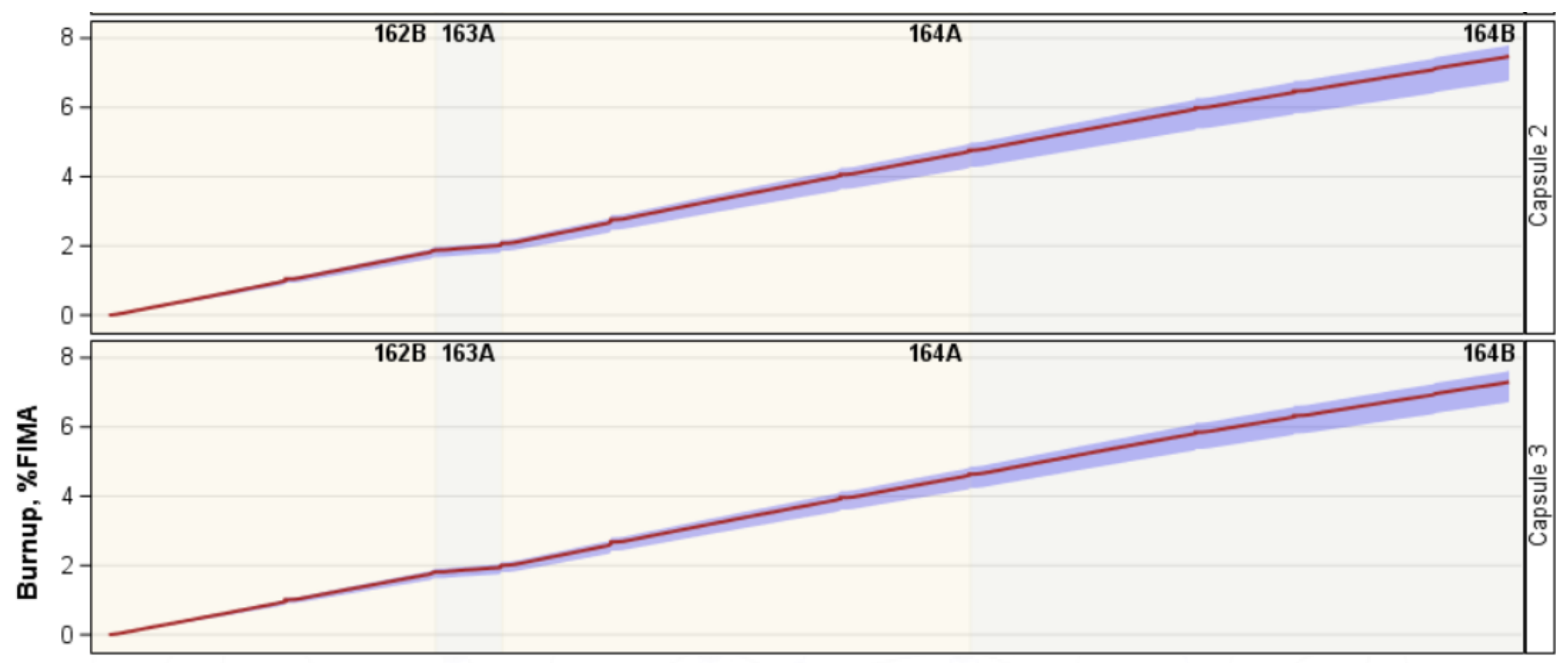




\section{AGR-5/6/7 Irradiation Look Ahead}

\section{AGR-5/6/7 peak burnup by cycle and cumulative}

\begin{tabular}{|c|c|c|c|c|c|c|c|c|c|c|c|c|c|c|c|c|}
\hline \multicolumn{8}{|c|}{ Original Estimate from ECAR-2961 } & \multicolumn{8}{|c|}{ Actual Followed by Projected } & \\
\hline Cycle & Cycle & Cycle & Lobe & Lobe & Cum Lobe & & Peak Burnup & Cycle & Cycle & Lobe & Lobe & \begin{tabular}{|c|c|}
$\begin{array}{c}\text { Cumulativ } \\
\text { Lobe }\end{array}$ \\
\end{tabular} & & \begin{tabular}{|l|} 
Proj Cycle \\
Burnup \\
\end{tabular} & Peak Burnup & \\
\hline \multirow[t]{2}{*}{ No. } & \multirow[t]{2}{*}{ Type } & \multirow{2}{*}{$\begin{array}{l}\text { Length } \\
\text { (EFPD) }\end{array}$} & \multirow{2}{*}{$\begin{array}{l}\text { Power } \\
\text { (MW) }\end{array}$} & \multirow{2}{*}{$\begin{array}{l}\text { Output } \\
\text { (MWD) }\end{array}$} & Output & & & & \multirow{2}{*}{$\begin{array}{l}\text { Length } \\
\text { (EFPD) }\end{array}$} & \multirow{2}{*}{\begin{tabular}{|c|} 
Power \\
(MW)
\end{tabular}} & Output & Output & & & & \\
\hline & & & & & (MWD) & Filter & (\%) & & & & (MWD) & (MWD) & Filter & $(\%)$ & $(\%)$ & \\
\hline 1 & Regular & 50 & 14 & 700 & 700 & $\mathbf{M}$ & 2.5 & Reg 162B & 43 & 14.25 & 613 & 613 & M & & 1.95 & \\
\hline 2 & Regular & 50 & 14 & 700 & 1400 & M & 4.9 & PALM 163A & 9 & 7.2 & 65 & 678 & $M$ & & 2.0 & \multirow[t]{5}{*}{ ACTUAL } \\
\hline 3 & PALM & 15 & 20 & 300 & 1700 & $\mathrm{H}$ & 5.6 & Reg 164A & 57 & 15.44 & 880 & 1558 & $\mathbf{M}$ & & 5.0 & \\
\hline 4 & Regular & 50 & 14 & 700 & 2400 & $\mathbf{M}$ & 7.5 & Reg 164B & 65 & 16.11 & 1047 & 2605 & M & & 7.8 & \\
\hline 5 & Regular & 50 & 14 & 700 & 3100 & $\mathbf{M}$ & 9.3 & PALM 165A & 14 & 19 & 266 & 2871 & $\mathbf{M}$ & 0.62 & 8.4 & \\
\hline 6 & PALM & 15 & 20 & 300 & 3400 & $\mathbf{M}$ & 10.0 & Reg $166 \mathrm{~A}$ & 59 & 17 & 1003 & 3874 & $\mathbf{L}$ & 2.44 & 10.9 & \\
\hline 7 & Regular & 50 & 14 & 700 & 4100 & $\mathbf{L}$ & 11.7 & Reg 166B & 60 & 17 & 1020 & 4894 & $\mathbf{L}$ & 2.04 & 12.9 & PROJECTED \\
\hline 8 & Regular & 50 & 16 & 800 & 4900 & $\mathbf{L}$ & 13.3 & PALM 167A & 6 & 20 & 120 & 5014 & $\mathbf{L}$ & 0.24 & 13.1 & \\
\hline 9 & PALM & 15 & 20 & 300 & 5200 & $\mathbf{L}$ & 13.9 & Reg 168A & 60 & 21 & 1260 & 6274 & $\mathbf{L}$ & 2.24 & 15.4 & Cycle end \\
\hline 10 & Regular & 50 & 18 & 900 & 6100 & $\mathbf{L}$ & 15.5 & Reg 168B & 60 & 21 & 1260 & 7534 & $\mathbf{L}$ & 1.96 & 17.3 & July 2020 \\
\hline 11 & Regular & 50 & 18 & 900 & 7000 & $\mathbf{L}$ & 16.9 & PALM $169 \mathrm{~A}$ & 14 & 21 & 294 & 7828 & $\mathbf{L}$ & 0.37 & 17.7 & Nov 2020 \\
\hline 12 & PALM & 15 & 20 & 300 & 7300 & $\mathbf{L}$ & 17.3 & Reg 170A & 60 & 21 & 1260 & 9088 & $\mathbf{L}$ & 1.58 & 19.3 & Feb 2021 \\
\hline 13 & Regular & 50 & 18 & 900 & 8200 & $\mathbf{L}$ & 18.5 & Total & 507 & & & & & & & \\
\hline \multirow[t]{2}{*}{ Total } & & 510 & & 8,200 & 8200 & & & & & \multirow{2}{*}{\multicolumn{4}{|c|}{$\begin{array}{l}21 \mathrm{MW} \text { lobe powers are not currently in the ISOP. } \\
\text { Would need to be requested. }\end{array}$}} & & & \\
\hline & & & & & & & & & & & & & & & & \\
\hline
\end{tabular}




\section{AGR-5/6/7 Irradiation Look Ahead}

Actual Followed by Projected

\begin{tabular}{|c|c|c|c|c|c|c|c|c|}
\hline Cycle & Cycle & Lobe & Lobe & $\begin{array}{l}\text { Cumulativ } \\
\text { Lobe }\end{array}$ & & \begin{tabular}{|l|} 
Proj Cycle \\
Burnup
\end{tabular} & Peak Burnup & \\
\hline & Length & Power & Output & Output & & & & \\
\hline & (EFPD) & $(\mathrm{MW})$ & (MWD) & (MWD) & Filter & $(\%)$ & $(\%)$ & \\
\hline Reg 162B & 43 & 14.25 & 613 & 613 & $\mathbf{M}$ & & 1.95 & \\
\hline PALM 163A & 9 & 7.2 & 65 & 678 & $M$ & & 2.0 & ACTUAL \\
\hline $\operatorname{Reg} 164 \mathrm{~A}$ & 57 & 15.44 & 880 & 1558 & $M$ & & 5.0 & \\
\hline Reg 164B & 65 & 16.11 & 1047 & 2605 & $\mathbf{M}$ & & 7.8 & \\
\hline PALM 165A & 14 & 19 & 266 & 2871 & $M$ & 0.62 & 8.4 & \\
\hline $\operatorname{Reg} 166 \mathrm{~A}$ & 59 & 17 & 1003 & 3874 & $\mathbf{L}$ & 2.44 & 10.9 & \\
\hline Reg 166B & 60 & 17 & 1020 & 4894 & $\mathbf{L}$ & 2.04 & 12.9 & PROJECTED \\
\hline PALM 167A & 6 & 20 & 120 & 5014 & $\mathbf{L}$ & 0.24 & 13.1 & \\
\hline $\operatorname{Reg} 168 \mathrm{~A}$ & 60 & 21 & 1260 & 6274 & $\mathbf{L}$ & 2.24 & 15.4 & Cycle end \\
\hline Reg 168B & 60 & 21 & 1260 & 7534 & $\mathbf{L}$ & 1.96 & 17.3 & July 2020 \\
\hline PALM 169A & 14 & 21 & 294 & 7828 & $\mathbf{L}$ & 0.37 & 17.7 & Nov 2020 \\
\hline $\operatorname{Reg} 170 \mathrm{~A}$ & 60 & 21 & 1260 & 9088 & $L$ & 1.58 & 19.3 & Feb 2021 \\
\hline \multirow[t]{3}{*}{ Total } & 507 & & & & & & & \\
\hline & & \multirow{2}{*}{\multicolumn{4}{|c|}{$\begin{array}{l}21 \mathrm{MW} \text { lobe powers are not currently in the ISOP. } \\
\text { Would need to be requested. }\end{array}$}} & & & \\
\hline & & & & & & & & \\
\hline
\end{tabular}




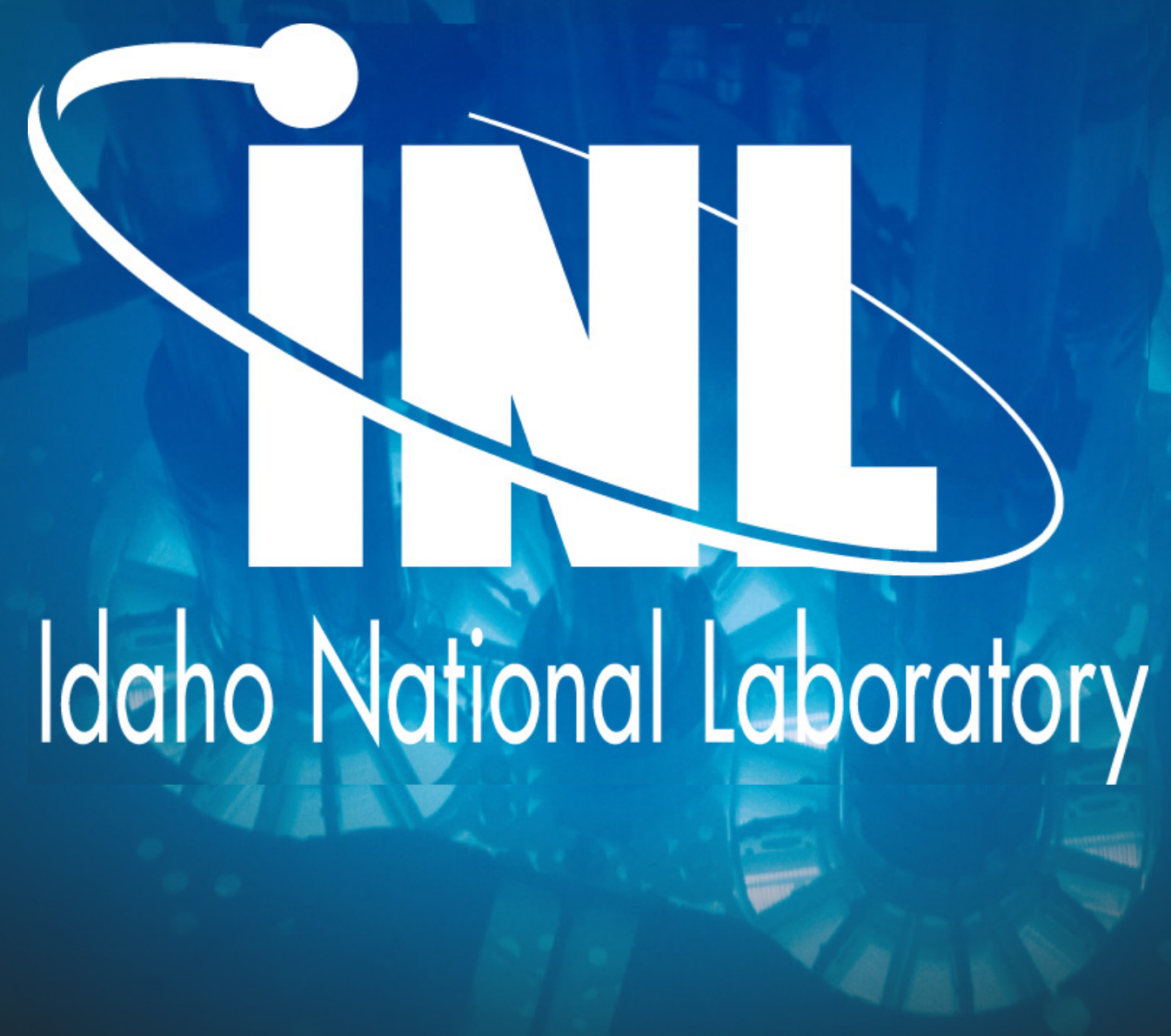

